\title{
Phase-Space Explorations in Time-Dependent Density Functional Theory
}

\author{
A. K. Rajam \\ Department of Physics, The Graduate Center of the City University of New York, 365 Fifth Ave, New York, NY 10016, USA \\ Paul Hessler and Christian Gaun \\ Department of Physics and Astronomy, Hunter College and City \\ University of New York, 695 Park Avenue, New York, NY 10065, USA \\ Neepa T. Maitra \\ Department of Physics, The Graduate Center of the City University of New York, \\ 365 Fifth Ave, New York, NY 10016, USA and \\ Department of Physics and Astronomy, Hunter College and City \\ University of New York, 695 Park Avenue, New York, NY 10065, USA
}

(Dated: December 14, 2018)

\begin{abstract}
We discuss two problems which are particularly challenging for approximations in time-dependent density functional theory (TDDFT) to capture: momentum-distributions in ionization processes, and memory-dependence in real-time dynamics. We propose an extension of TDDFT to phasespace densities, discuss some formal aspects of such a "phase-space density functional theory" and explain why it could ameliorate the problems in both cases. For each problem, a two-electron model system is exactly numerically solved and analysed in phase-space via the Wigner function distribution.
\end{abstract}

PACS numbers:

\section{INTRODUCTION}

Time-dependent density functional theory (TDDFT) is a remarkably successful theory of many-body systems in time-dependent external potentials $[1-3]$. The analog of static density functional theory [4], TDDFT is based on the Runge-Gross proof of a one-to-one mapping between the external potential and the time-dependent density of electrons evolving under it, for a specified initial wavefunction [1]. Because knowledge of the external potential specifies the many-body Hamiltonian, all properties of the interacting electronic system can be extracted from just its one-body density and the initial-state. In practise, TDDFT utilizes the Kohn-Sham (KS) scheme, where the time-dependent density of an interacting system evolving in an external potential $v_{\text {ext }}(\mathbf{r}, t)$ is calculated from a fictitious noninteracting system of fermions moving in an effective potential, the KS potential $v_{\mathrm{S}}(\mathbf{r}, t)$, that is defined to reproduce the density of the interacting system. The KS potential is written as the sum:

$v_{\mathrm{S}}\left[n ; \Phi_{0}\right](\mathbf{r}, t)=v_{\mathrm{ext}}(\mathbf{r}, t)+v_{\mathrm{H}}[n](\mathbf{r}, t)+v_{\mathrm{XC}}\left[n ; \Psi_{0}, \Phi_{0}\right](\mathbf{r}, t)$

where $n(\mathbf{r}, t)$ denotes the time-dependent density, and $\Psi_{0}\left(\Phi_{0}\right)$ is the initial interacting(noninteracting) wavefunction. The second term, $v_{\mathrm{H}}[n](\mathbf{r}, t)=\int \frac{n\left(\mathbf{r}^{\prime}, t\right)}{\left|\mathbf{r}-\mathbf{r}^{\prime}\right|} d^{3} r^{\prime}$, is the classical Hartree potential, while the third is the exchange-correlation (xc) potential $v_{\mathrm{xC}}$. This is unknown as a functional of the time-dependent density and initialstates, and must be approximated in practise. Armed with an approximation for $v_{\mathrm{XC}}\left[n ; \Psi_{0}, \Phi_{0}\right](\mathbf{r}, t)$, one then propagates the time-dependent $\mathrm{KS}$ equation:

$$
i \partial_{t} \phi_{i}(\mathbf{r}, t)=\left(-\nabla^{2} / 2+v_{\mathrm{S}}(\mathbf{r}, t)\right) \phi_{i}(\mathbf{r}, t)
$$

finding the $N$ single-particle orbitals $\phi_{i}(\mathbf{r}, t)$, that initially made up the initial KS determinant $\Phi_{0}$. (Atomic units, $e^{2}=\hbar=m_{e}=1$ are used throughout this paper). The density of the interacting system is obtained from $n(\mathbf{r}, t)=\sum_{i}^{N}\left|\phi_{i}(\mathbf{r}, t)\right|^{2}$, and the Runge-Gross theorem then assures us that all properties of the true system can be extracted from the orbitals. In the linear response regime, in which lie the majority of TDDFT applications so far, excitation energies and oscillator strengths are obtained from a perturbative formulation of these equations $[5,6]$, leading to the matrix formulation that operates in most of the quantum chemistry codes today.

Clearly the accuracy of TDDFT then depends on the approximation used for the xc kernel. Despite being known generally to have "memory-dependence" - that is, $v_{\mathrm{xC}}(\mathbf{r}, t)$ depends on the history $n\left(\mathbf{r}, t^{\prime}\right), t^{\prime} \leq t$ and on the initial-states, $\Psi_{0}$ and $\Phi_{0}[7-9]$ - almost all approximations used today neglect this, and bootstrap a ground-state functional: that is, the instantaneous density is input into an approximate ground-state functional, $v_{\mathrm{xC}}^{\mathrm{A}}\left[n ; \Psi_{0}, \Phi_{0}\right](\mathbf{r}, t)=v_{\mathrm{XC}}^{\mathrm{gs}}[n(\mathbf{r} t)](\mathbf{r} t)$. The superscript A denotes this "adiabatic approximation". In the linear response regime $[5,6]$, adiabatic TDDFT has achieved an unprecedented balance between accuracy and efficiency for the calculations of excitations and response properties. For the first time, one can compute the quantum mechanical spectrum of systems as large as biomolecules (see eg. Refs. [10-12]), run coupled electron-ion dynamics on interesting chemical reactions [13], and study molecular transport through nanostructures [14]. Excitations for which the adiabatic approximation fails have been recognized, and, in various stages of being corrected [1518]. Sometimes the spatial long-ranged-ness of the true 
functional is important, and approximations more sophisticated than the usual semi-local ones are required [1922].

But TDDFT applies also for strong field dynamics, and this is the regime it is particularly promising for, given that correlated wavefunction calculations for more than two or three electrons in strong-fields become prohibitively expensive [23]. Compared to calculations of spectra, in addition to an approximation for the xc potential, now a new ingredient needs to be considered in the calculation: observable functionals, i.e. the observables of interest must be expressed as functionals of the KS wavefunction. The RG theorem guarantees that all properties of the true system can be obtained from the KS orbitals, as they themselves are implicit density-initialstate-functionals, but how? If the property is related directly to the density, then no additional observable functional is needed. For example, in high-harmonicgeneration it is the dipole moment, $\mathbf{d}=\int n(\mathbf{r}, t) \mathbf{r} d^{3} r$ that is of interest, and indeed TDDFT calculations have been successful for high-harmonic generation spectra of a range of interesting systems [24-26]. But if we are interested in, for example, measuring double-ionization probabilities, we require the interacting pair-density as a functional of the density (or KS orbitals), and this is highly non-trivial [27]. Non-sequential double-ionization is a fascinating problem that dogged TDDFT for several years [27-29]: TDDFT's promise in capturing electron correlation made it attractive for this problem, but it was soon realized that not only does one need to go beyond the usual semilocal GGA's for the xc functional in order to obtain the knee structure [29], but using an uncorrelated KS expression for the pair-density yielded a knee that was too high. (In Ref. [27], an adiabatic correlated expression, based on the ground-state PerdewWang pair density [30] was shown to lower the knee appropriately). Approximating a general observable simply by the appropriate operator evaluated on the KS wavefunction can lead to gross inaccuracies, due to the lack of correlation in the KS wavefunction itself. It is important to remember that the KS wavefunction is designed to reproduce the exact interacting one-body density, but is not supposed to be an approximation to the true wavefunction. Indeed another aspect of the same doubleionization problem recently demonstrated this: Wilken and Bauer's calculations of ion-momentum-recoil distributions in double-ionization [31] showed that the KS momentum-distributions were drastically wrong, displaying a single maximum instead of the characteristic twohump structure, and with a significantly overestimated magnitude. Typically KS momentum distributions are not those of the true system, even if the exact KS orbitals were used, that is if the exact $v_{\mathrm{XC}}$ was known for the problem, and used to generate the KS orbitals. Another example of this will be given in Sec. III A.

Returning to the question of the approximate $v_{\mathrm{xC}}\left[n ; \Psi_{0}, \Phi_{0}\right](\mathbf{r}, t)$ : the accuracy of the adiabatic approximation for real-time dynamics gets mixed reviews. In strong-field double-ionization, the xc potential appears not to be significantly non-local in time in a wide range of cases, although it depends on how the field is ramped on [32]. Dynamics of two electrons in parabolic wells, on the other hand, yield an exact correlation potential that appear to depend strongly on the history [33-35]. We shall revisit Ref. [35] in Sec. III B. Although significant advances have been made in understanding $[7,8]$ and modelling [34, 36-40] memory-dependence, it remains today a difficult problem.

One situation in which the usual approximations (spatially semi-local and adiabatic density dependence) do poorly, is when the true wavefunction fundamentally cannot be described by a single Slater-determinant. This is also true for the ground state where molecular dissociation curves are a notorious problem [41-43]: when an electron-pair bond dissociates, the true wavefunction develops a Heitler-London character, which in a minimal description requires two Slater-determinants. The KS description however operates via a single Slaterdeterminant. The exact xc potential allows for the fundamental difference in the KS and true wavefunctions by developing rather stark and unusual peak and step features, that are difficult to capture in approximations. In time-dependent problems, coefficients of the interacting wavefunction expanded in a basis of single-Slaterdeterminants may change in time dramatically: whereas at one time a single-Slater determinant may dominate, at a later time, two single-Slater determinants are essential. Such situations may happen in electronic quantum control problems (now becoming experimentally accessible with the advent of attosecond lasers) [44]. Consider for example evolving the ground-state of the He atom $\left(1 \mathrm{~s}^{2}\right)$ to its first accessible excited state $(1 \mathrm{~s} 2 \mathrm{p})$. The KS ground state is a single-slater determinant composed of a doubly-occupied spatial orbital. This evolves under the KS Hamiltonian, which is a one-body evolution operator. The target single excitation is however a double Slater determinant, and therefore cannot be reached by any one-body operator. The exact KS system attains the density of the true target state using a single orbital, not two. This yields a very unnatural description of the true system; consequently, the KS potential develops rather unnatural features, difficult to model $[8,9]$. The inability of KS methods to change occupation numbers leads to challenges in developing approximations.

The two ingredients needed in a TDDFT calculation - approximations for the xc potential and the relevant observable functional - determine the accuracy of the results. As discussed above, sometimes the simplest approximations work well, but in other cases, it is more challenging to derive suitable approximations. In this paper, we explore a generalization of TDDFT based on the phase-space density. That is, instead of taking the coordinate-space density, $n(\mathbf{r}, t)$ as our basic variable, we consider taking the density in phase-space, $w(\mathbf{r}, \mathbf{p}, t)$ : a quasi-probability distribution which is somewhat related to the probability of finding an electron at position $\mathbf{r}$ 
with momentum $\mathbf{p}$ (see Sec. II). The idea here is twofold: First, as the basic variable has more information, functionals of them may be simpler to approximate, or equally, simple functionals of them may be more accurate than simple functionals of the coordinate-density alone, while retaining the favorable system-size scaling property of density functional theories. Second, more observables are directly obtained without needing additional observable-functionals, in particular, those pertaining to one-body operators, such as momentum, kinetic energy. As the coordinate and momentum operators do not commute, there is no unique phase-space density function, and we begin in Sec. II by discussing the Wigner function, which turns out to be a particularly useful choice for our purposes. We discuss the 1-1 mapping between Wigner functions and potentials, for a given initial-state. We discuss also the formal equivalence of the Wigner function with time-dependent density-matrix functional theory, which has seen a recent resurgence of interest [4547]. Next, we return to TDDFT and explore the true and KS phase-spaces, and the implications of a phasespace based theory, for two examples pertaining to the challenges in TDDFT described above: momentum distributions (Sec. III A) and history-dependence in the xc functional (Sec IIIB).

\section{THE 1-BODY WIGNER PHASE-SPACE DENSITY}

Quantum phase-space distributions have played an important role in the development of quantum mechanics. Although a precise joint probability distribution in position and momentum is impossible due to the Heisenberg uncertainty principle, various quasi-probability distributions have been defined, which contain position and momentum distribution information in a way consistent with the uncertainty principle. Such distributions are particularly useful in a semiclassical context, relating the quantum states to the underlying classical trajec- tories, and have been exploited extensively, for example in quantum chaos and quantum optics. Phase-space approaches have however been largely, although not entirely [48-53] neglected in density functional theories, where position plays a preferred role over momentum. Most recently [51-53], Gill and co-workers, have developed and tested models for the ground-state correlation energy based on various "phase-space intracules" which are essentially different contractions of the two-body reduced Wigner function. For example, there is the Omega intracule, which is a function of three variables; the distance between two electrons, the magnitude of the difference in their momentum vectors, and the angle between their position and momentum [53]. This is motivated by the very physical idea that the correlation between two electrons depends on their relative momentum as well as on their relation distance [54].

Wigner functions were first introduced in 1932 [5557 , with the intent of application to many-body systems. The Wigner function quasi-probability distributions are real but not positive semi-definite; indeed the negative areas have been interpreted as an indication of non-classical behaviour, from interference to tunneling processes, and research in how to classify these areas continues today (see for example Refs. [58]). Although initially introduced to treat many-particle dynamics, the majority of applications of the Wigner function actually involve one-particle systems, especially for making quantum-classical correspondence. It has been argued that the Wigner function is a particularly suitable approach to studying transport [59-61] due to its setting within a semiclassical picture while yet being rigorously quantum mechanical. Ref. [61] develops a formalism based on reduced-Wigner-functions for indistinguishable fermionic systems accounting for Pauli-exchange (but not correlation).

For a system of $N$ identical particles, the reduced $k$ body Wigner phase space density is defined as the Fourier transform of the $k$ th-order density matrix $\rho_{k}$ :

$$
w_{k}\left(\mathbf{r}_{1} . . \mathbf{r}_{k}, \mathbf{p}_{1} . . \mathbf{p}_{k}, t\right)=\left(\frac{1}{2 \pi}\right)^{3 k} \int d^{3} y_{1} . . d^{3} y_{k} e^{i \sum_{k} \mathbf{p}_{\mathbf{k}} \cdot \mathbf{y}_{\mathbf{k}}} \rho_{k}\left(\mathbf{r}_{\mathbf{1}}-\mathbf{y}_{\mathbf{1}} / 2 . . \mathbf{r}_{\mathbf{k}}-\mathbf{y}_{\mathbf{k}} / 2, \mathbf{r}_{\mathbf{1}}+\mathbf{y}_{\mathbf{1}} / 2 \ldots \mathbf{r}_{\mathbf{k}}+\mathbf{y}_{\mathbf{k}} / 2 ; t\right)
$$

$$
\begin{aligned}
& \text { where the } k \text {-th order density ma- (henceforth referred to as simply Wigner function): } \\
& \begin{array}{ccr}
\operatorname{trix} & \text { is } \quad \rho_{k}\left(\mathbf{r}_{\mathbf{1}}^{\prime} . . \mathbf{r}_{\mathbf{k}}^{\prime}, \mathbf{r}_{1} . . \mathbf{r}_{2} ; t\right) & = \\
\left(\begin{array}{c}
N \\
k
\end{array}\right) \int \Psi^{*}\left(\mathbf{r}_{1}^{\prime} . . \mathbf{r}_{N}^{\prime}, t\right) \Psi\left(\mathbf{r}_{1} . . \mathbf{r}_{N}, t\right) d r_{k+1} . . d r_{N} . & \text { We }
\end{array} \\
& \text { consider here a density-functional theory based on } \\
& \text { the one-body Wigner function } w(\mathbf{r}, \mathbf{p}, t) \equiv w_{1}(\mathbf{r}, \mathbf{p}, t) \\
& w(\mathbf{r}, \mathbf{p}, t)=\left(\frac{1}{2 \pi}\right)^{3} \int d^{3} y \rho_{1}(\mathbf{r}-\mathbf{y} / 2, \mathbf{r}+\mathbf{y} / 2, t) e^{i \mathbf{p} \cdot y}
\end{aligned}
$$


momentum-space are directly obtained by integration:

$$
\begin{aligned}
n(\mathbf{r}, t) & =\int w(\mathbf{r}, \mathbf{p}, t) d^{3} p \\
\tilde{n}(\mathbf{p}, t) & =\int w(\mathbf{r}, \mathbf{p}, t) d^{3} r
\end{aligned}
$$

Several observations can immediately be made about such a phase-space density-functional theory (PSDFT) whose basic variable is the Wigner phase-space density (taking the role of the coordinate-space density in usual density functional theories). First, the theory is formally equivalent to the recently developed time-dependent onebody density-matrix functional theory (1DMFT) [45-47]: $w$ and $\rho_{1}$ contain exactly the same information, in different forms related via a Fourier transform. Second, as in the 1DMFT, there is no Kohn-Sham counterpart: the Wigner-function of an interacting system cannot be reproduced by any non-interacting system in a local potential. In a non-interacting system, the one-body reduced density matrix is idempotent, while in an interacting system, it cannot be. It is interesting that generally while a non-interacting system may be found that reproduces the coordinate-space density of an interacting system, and a different non-interacting system may be found reproducing its momentum-space density, one cannot find a non-interacting system that reproduces its phase-space density. Third, the Wigner function directly gives the expectation value of any one-body operator: no additional observable functional would be needed, for example, for kinetic energy or momentum distributions. Fourth, the one-to-one mapping between the potential and the phase-space density, for a given initial-state, follows directly from the Runge-Gross theorem: We have

$$
\begin{array}{r}
w(r, p, t) \rightarrow n(r, t) \\
\left(n(\mathbf{r}, t), \Psi_{0}\right) \rightarrow \Psi\left(\mathbf{r}_{1} . . \mathbf{r}_{N}, t\right)
\end{array}
$$

where both arrows indicate a unique mapping, the first following from $p$-integration (Eq. 5), and the second from $\mathrm{RG}$, while also

$$
\Psi\left(\mathbf{r}_{1} . . \mathbf{r}_{N}, t\right) \rightarrow w(r, p, t)
$$

uniquely for a specified initial-state. Hence, for local external potentials, there is a 1-1 mapping between the onebody Wigner function and the many-body wavefunction for a given initial state, i.e. that all observables may be extracted from the one-body Wigner function and initialstate alone.

We note that this 1-1 mapping holds for local external potentials (i.e. purely multiplicative in coordinate-space. Gilbert [62] has shown that for the ground state, the extension of the Hohenberg-Kohn therem to one-body reduced density matrices applies also to the wider class of non-local potentials, $v\left(\mathbf{r}, \mathbf{r}^{\prime}\right)$. Formally, one may be led to search for a one-to-one $\rho_{1}-v\left(\mathbf{r}, \mathbf{r}^{\prime}\right)$ mapping in the time-dependent case also, given that these are conjugate variables that couple together in an energy functional, (c.f. the $n-v$ mapping of Runge-Gross or HohenbergKohn [4], and the $A-j$ mapping of Ghosh and Dhara in TD-current-density functional theory [20,63]. Although a proof for time-dependent non-local potentials is yet to be found, the mapping may be generalized to vector potentials $\mathbf{A}(\mathbf{r}, t)$, extending the realm of application to include magnetic fields:

$$
\begin{array}{r}
w \rightarrow \mathbf{j}(\mathbf{r}, t) \\
\left(\mathbf{j}(\mathbf{r}, t), \Psi_{0}\right) \rightarrow \Psi\left(\mathbf{r}_{1} . . \mathbf{r}_{N}, t\right)
\end{array}
$$

where the first arrow follows from

$$
\mathbf{j}(\mathbf{r}, t)=\int d^{3} \mathbf{p} w(\mathbf{r}, \mathbf{p}, t)
$$

and the second from the Ghosh-Dhara proof [63] of the 11 mapping between currents and vector-potentials. That is, any observable of any time-dependent interacting electronic system evolving under an external vector potential, is a functional of its initial wavefunction and timedependent one-body phase-space density.

The Runge-Gross proof proceeded in two steps, where the first proved a one-to-one mapping between currents and (scalar) potentials for a given initial state.The second step, to go from currents to densities, holds under the condition that a boundary term, that involves the density and the gradient of the potential, vanishes. The implications of this boundary condition have been discussed in Ref. [64]; it means that TDDFT cannot be applied to periodic systems in uniform electric fields [65]. We point out that here there is no requirement on the boundary conditions for the $w-v$ (or $w-\mathbf{A}$ mappings to hold.

Time-dependent phase-space functional theory involves solving the equation of motion for the Wigner function:

$$
\begin{aligned}
\dot{w}(\mathbf{r}, \mathbf{p}, t) & =\left[-\mathbf{p} \cdot \nabla-i \int d^{3} p^{\prime} \int d^{3} y e^{-i\left(\mathbf{p}-\mathbf{p}^{\prime}\right) \cdot \mathbf{y}}\left[v_{\text {ext }}\left(\mathbf{r}+\frac{\mathbf{y}}{\mathbf{2}}\right)-v_{\text {ext }}\left(\mathbf{r}-\frac{\mathbf{y}}{\mathbf{2}}\right)\right]\right] w(\mathbf{r}, \mathbf{p}, t) \\
& -i \int d^{3} y d^{3} p_{1} d^{3} p_{2} d^{3} r_{2} e^{i\left(\mathbf{p}-\mathbf{p}_{1}\right) \cdot y}\left(\frac{1}{\left|\mathbf{r}-\mathbf{r}_{2}+\frac{y}{2}\right|}-\frac{1}{\left|\mathbf{r}-\mathbf{r}_{2}-\frac{y}{2}\right|}\right) w_{2}\left(\mathbf{r}, \mathbf{r}_{2}, \mathbf{p}_{1}, \mathbf{p}_{2}, t\right)
\end{aligned}
$$




$$
i \dot{\rho}_{1}\left(\mathbf{r}^{\prime}, \mathbf{r}, t\right)=\left(-\nabla^{2} / 2+v_{\mathrm{ext}}(\mathbf{r}, t)+\nabla^{\prime 2} / 2-v_{\mathrm{ext}}\left(\mathbf{r}^{\prime}, t\right)\right) \rho_{1}\left(\mathbf{r}^{\prime}, \mathbf{r}, t\right)+\int d^{3} r_{2}\left(\frac{1}{\left|\mathbf{r}-\mathbf{r}_{\mathbf{2}}\right|}-\frac{1}{\left|\mathbf{r}^{\prime}-\mathbf{r}_{\mathbf{2}}\right|}\right) \rho_{2}\left(\mathbf{r}^{\prime}, \mathbf{r}_{2}, \mathbf{r}, \mathbf{r}_{2}, t\right)
$$

where $\rho_{2}$ is the second-order density-matrix, to be approximated as a functional of $\rho_{1}$. This equation is first in the BBGKY (Bogoliubov-Born-Green-Kirkwood-Yvon) heirarchy of reduced density matrix evolutions, which gives the equation of motion for the $k$ th reduced DM in terms of the $(k+1)$ st and lower DM's. Eq. II is the crux of $1 \mathrm{DMFT}$, which has only very recently begun to be explored. All the development and results so far have been in the linear response regime [45-47], using functionals for $\rho_{2}$ which have been adiabatically bootstrapped from ground-state ones (eg. Refs.[66, 67]).

The accuracy of the time-dependent PSDFT(1DMFT) depends on approximate functionals for the term involving the second-order Wigner function $w_{2}\left(\rho_{2}\right)$ in terms of $w\left(\rho_{1}\right)$. As has been noted above, and earlier in the literature, the kinetic term is treated exactly, in contrast to DFT, where the correlation potential/energy has a kinetic component. The simplest approximation would be the uncorrelated one, where $\rho_{2}$ is written as a product of antisymmetrized one-body $\rho_{1}$ 's. This truncates the BBGKY heirarchy at the first level, and is equivalent to time-dependent Hartree-Fock. Its inability to change occupation numbers makes it unable to treat many of the challenges that TDDFT faces, that the phase-space DFT would hope to treat. For example, the electronic quantum control problem mentioned in the introduction (see also [68]). Section III A discusses another example where a change in occupation number is crucial for an accurate description. Natural orbitals $\psi_{i}(\mathbf{r}, t)$ with occupations $f_{i}(t)$ are defined by diagonalizing $\rho_{1}\left(\mathbf{r}, \mathbf{r}^{\prime}, t\right)$ :

$$
\rho_{1}\left(\mathbf{r}, \mathbf{r}^{\prime}, t\right)=\sum_{i} f_{i}(t) \psi_{i}(\mathbf{r}, t) \psi_{i}^{*}\left(\mathbf{r}^{\prime}, t\right)
$$

We may define "natural Wigner orbitals" via the analogous expansion:

$$
w(\mathbf{r}, \mathbf{p}, t)=\sum_{i} f_{i}(t) w_{i}(\mathbf{r}, \mathbf{p}, t)
$$

where $w_{i}(\mathbf{r}, \mathbf{p}, t)=\int d^{3} y \phi_{i}^{*}(\mathbf{r}-y / 2) \phi_{i}(\mathbf{r}+y / 2, t)$. From Eq. II the time evolution of the occupation numbers can be derived:

$$
\begin{array}{r}
i \dot{f}_{i}(t)=\iiint \frac{1}{\left|\mathbf{r}^{\prime}-\mathbf{r}_{\mathbf{2}}\right|} \rho_{2}\left(\mathbf{r}, \mathbf{r}_{\mathbf{2}}, \mathbf{r}^{\prime}, \mathbf{r}_{\mathbf{2}}, t\right) \\
\phi_{i}^{*}(\mathbf{r}, t) \phi_{i}\left(\mathbf{r}^{\prime}, t\right) d \mathbf{r} d \mathbf{r}^{\prime} d \mathbf{r}_{\mathbf{2}}-c . c
\end{array}
$$

The right-hand-side of this equation is only nonzero if the contraction of $\rho_{2}$ on to the natural orbitals, $\phi_{i}^{*}(\mathbf{r}, t) \rho_{2}\left(\mathbf{r}, \mathbf{r}_{\mathbf{2}}, \mathbf{r}^{\prime}, \mathbf{r}_{\mathbf{2}}, t\right) \phi_{i}\left(\mathbf{r}^{\prime}, t\right)$, is imaginary. Therefore occupation numbers cannot change with using an uncorrelated product expression for $\rho_{2}$. It was also shown that bootstrapping any adiabatic functional from ground-state 1DMFT cannot change occupation numbers [47, 69]. A systematic approach might consider then truncating the BBGKY heirarchy at the next order; that is, solving the equation of motion for $\rho_{2}$ consistently with Eq. II, but using the uncorrelated (antisymmetrized product form) for $\rho_{3}$. However, it has been shown that this violates fundamental trace relations between density matrices of different order [70, 71]; ways to get around this become rapidly complicated. Moreover, one must deal with a four-point function (second-order Wigner function) rather than the two-point one (firstorder).

The problem of an appropriate $w_{2}$-functional in terms of $w$, which is able to change occupation numbers while retaining fundamental physical properties, is an important area for future research.

\section{REVISITING TWO CHALLENGES WITHIN PHASE-SPACE DFT}

We now describe in a little more detail two problems that are particularly challenging for TDDFT approximations, and discuss how a PSDFT can ameliorate them.

\section{A. Kohn-Sham momentum densities}

In general, the momentum-distribution of the KS system is not the same as that of the true system: although the sum of the squares of the KS orbitals in coordinatespace yield the exact density of the true system, the sum-square of their Fourier transforms to momentumspace does not yield the exact momentum-density. In cases where the correlation component to the kinetic energy, $T_{\mathrm{C}}$ is relatively small, calculating the momentum distribution simply from the Fourier transforms of the relevant KS orbitals can sometimes be quite accurate, as recently illustrated by the Compton profiles obtained in Ref. [72], provided accurate enough ground-state xc potentials are used. Earlier, for the the ground state case, Lam and Platzman derived a local-density approximation for the correlation correction to KS momentum- 
densities for Compton profiles [73]. The KS orbital momentum distribution has also been successful in electron momentum spectroscopy $[74,75]$ : this is essentially a triple differential cross-section measurement, where one electron is scattered from an atom, molecule, or surface, causing the ejection of another electron. The experiment obtains a map of the electron momentum distribution before ejection, thus imaging the momentum distribution of the Dyson orbital. Refs. [74, 75] show that the KS orbital yields a good approximation to the Dyson orbital in their momentum distributions, better than, for example, Hartree-Fock.

Away from the ground-state, Wilken and Bauer [31] have shown that KS momentum-distributions of TDDFT are miserably poor for non-sequential double-ionization processes; not only their shape is wrong, lacking the characteristic double-hump structure, but also their magnitude is significantly underestimated. Ref. [31] develops a "product-phase" approximation to extract the true momentum distribution from the KS system, i.e. an observable functional for the momentum-distribution.

The possibility of working directly in momentum-space is hindered by the lack of a one-to-one mapping theorem for momentum-densities (even for the ground-state case) $[77,78]$. In fact the earlier interest in developing ways to associate phase-space distributions to a given ground-state coordinate-space density [48-50] was motivated by trying to get good momentum-space properties derived from the coordinate-space density. One may view the density-matrix developments as a rigorous approach to this problem; as discussed in Sec. II, the exact one-body density matrix, or equivalently, the one-body phase-space density, directly yields all one-body information, i.e. the expectation value of any one-body operator, such as the momentum-density operator, is exact.

Even a simple model consisting of two electrons in one-dimension illustrates the discrepancy between the KS and true momentum-densities in ionization or scattering problems. With an exactly solvable model, one can pinpoint the origin of the differences between the momentum distributions of the true and KS systems. In this particular example, we will see it is due to the singleSlater determinant description being a poor description of a state in which two electrons are doing completely different things. One electon is at rest (modelling a "bound" electron), while the other is moving away from it (modelling a "scattered" or "ionized"electron), and we consider that enough time has elapsed that the true wavefunction describes independent electrons:

$$
\Psi\left(x, x^{\prime}, t\right)=\left(\psi_{0}(x) \psi_{p}\left(x^{\prime}, t\right)+\psi_{0}\left(x^{\prime}\right) \psi_{p}(x, t)\right) / \sqrt{2}
$$

The orbital for the bound electron is simply chosen as a Gaussian

$$
\psi_{0}(x)=e^{-x^{2} / 2} / \pi^{\frac{1}{4}}
$$

while that for the ionized electron is, for simplicity, chosen to be a free-particle dispersing Gaussian, which was initially ejected by some laser field (turned off thereafter), such that its initial spread in position is $1 / \Delta$, and average momentum is $p_{0}$ :

$$
\psi_{p}(x, t)=\sqrt{\frac{\Delta}{\sqrt{\pi\left(1+i t \Delta^{2}\right)}}} e^{\left(\frac{i p_{0} x-\Delta^{2} x^{2} / 2-i p_{0}^{2} t / 2}{1+i \Delta^{2} t}\right)}
$$

The coordinate-density, $n(x, t)=2 \int d x^{\prime}\left|\Psi\left(x, x^{\prime}, t\right)\right|^{2}$, and momentum-density, $\tilde{n}(p, t)$ = $2 \int d p^{\prime}\left|\int d x d x^{\prime} e^{i\left(p x+p^{\prime} x^{\prime}\right)} \Psi\left(x, x^{\prime}, t\right) /(2 \pi)\right|^{2}$ of this state at a snapshot in time is shown in Fig. 1: Neither are remarkable, as both are what might be expected quasiclassically from the sum of two probability distributions centered around the average position and momentum for each electron. Now we consider the Kohn-Sham description, which, for two electrons in a singlet state, operates via the doubly-occupied orbital, reproducing the time-evolving density $n(x, t)$ of the true system:

$$
\phi(x, t)=\sqrt{\frac{n(x, t)}{2}} \exp \left(i \int^{x} d x^{\prime} \frac{J\left(x^{\prime}, t\right)}{n\left(x^{\prime}, t\right)}\right)
$$

where $J(x, t)$ is the current-density of the true system. We compute the KS momentum distribution, $\tilde{n}_{\mathrm{S}}(p, t)=$ $|\tilde{\phi}(p, t)|^{2}$ from the Fourier transform $\tilde{\phi}$ of the orbital $\phi$. From Figure 1, we see that while the overall envelope of the KS momentum distribution follows that of the true system, there are distinct oscillations. In Figure 2 we plot the corresponding Wigner phase-space distributions, $w(x, p, t)$ and $w_{s}(x, p, t)$; oscillations are evident in the latter also, and dip into negative values. This is a typical signature of non-classical behavior: the KS orbital describes an electron spatially delocalized in two regions, impossible for a classical particle. The coherence between the two separated parts of the wavepacket gives rise to the oscillations in the phase-space density: integrating over momentum washes out these oscillations, yielding the sum of the two Gaussians in the left-handside of Fig. 1 (i.e the true interacting density) whereas the oscillations persist in the KS momentum distribution on the right. On the other hand, the true Wigner function looks much like a classical joint phase-space distribution would be: a peak at the position and momentum of each electron, each locally smeared out. Aside from the antisymmetrizing, the true wavefunction has classical features, with each electron in their independent Gaussian wavepackets, quite in contrast to the KS description which describes both electrons in the same delocalized orbital. Although yielding the exact coordinate density of the true system, this exact KS wavefunction is clearly far from the true wavefunction. Fundamentally, it associates to the true two-determinant state, a single determinant; in a sense, this is a time-dependent analog of the static correlation problems that haunt ground-state density functional theory, for example in dissociation problems $[16,41,76]$. We can understand the discrepancy in the momentum distributions as arising from this fundamental difference in the nature of the true and KS states 
in this particular case. (In the next section, we shall see an example where the momentum distributions are not so different). We stress that this is the KS system with the exact xc functional: what is required in TDDFT to get the correct exact momentum distribution from this, is the (unknown) momentum observable functional.
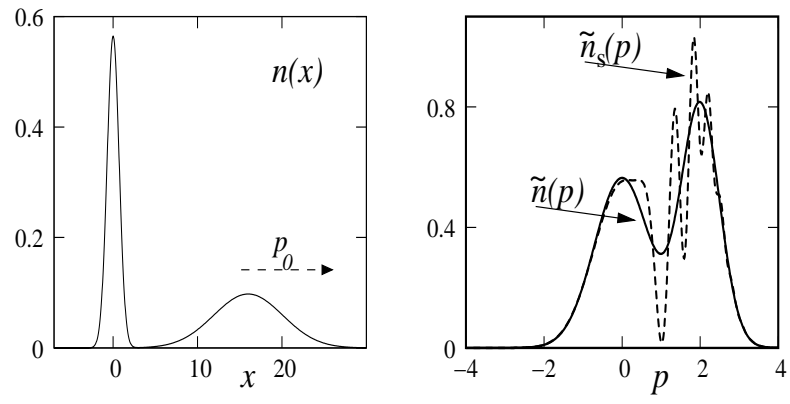

FIG. 1: Density profiles at time $t=8$, for parameters $\Delta=0.8$ and $p_{0}=2$. The left-hand panel shows the coordinate-density profile, identical by definition for KS and true. The right-hand panel shows the true (solid) and KS (dashed) momentumdensity profile.

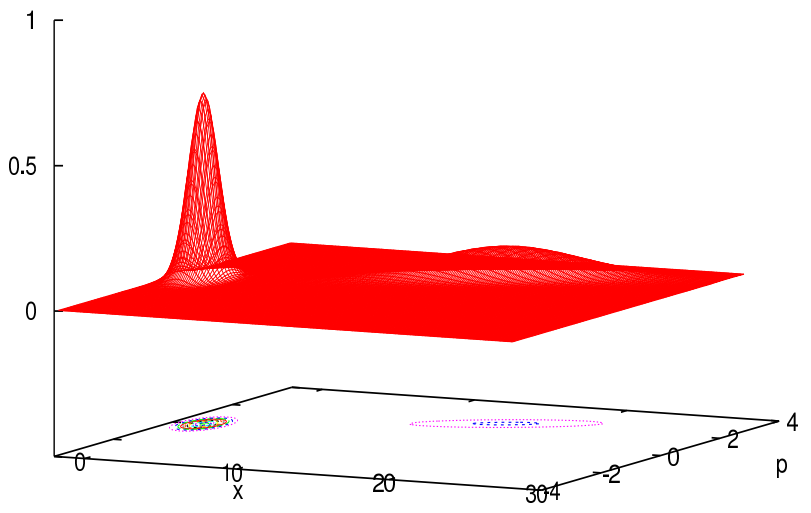

FIG. 2: The true Wigner profile at time $t=8$, for parameters $\Delta=0.8$ and $p_{0}=2$.

What are the implications of this for a PSDFT? As explained in the earlier section, in a phase-space DFT we deal directly with the interacting system; there is no non-interacting system that can reproduce an interacting phase-space density. Momentum distributions are immediately obtained without the need for an additional observable functional. And, had we the exact functional for the $w_{2}$-term in terms of $w$, these would be exact. In practise, approximations for this term are needed, and for this example, it is crucial for the approximation to be able to change occupation number. The state starts in a ground-state, both electrons bound, which, in a first approximation would be in the same doubly-occupied orbital.In the process of ionization, one electron leaves, and a two-orbital description is necessary, each with occupation number 0.5. Any approximation unable to change occupation numbers will once again yield wiggles in the momentum and phase-space distribution. These should

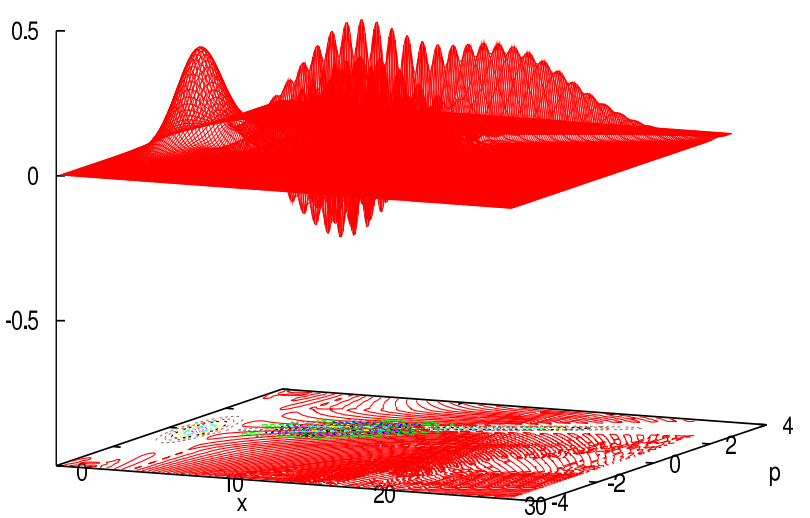

FIG. 3: The KS Wigner profile at time $t=8$, for parameters $\Delta=0.8$ and $p_{0}=2$.

not be there in such a state which is of classical nature. We comment here that a similar problem occurs for certain quantum control theory type problems, as discussed in the introduction.

\section{B. Dependence on the history of the density}

The time-dependent exchange-correlation potential functionally depends on the initial state (both the true and $\mathrm{KS})$ as well the history of the density $[1,8$, 9]. This dependence on the past is referred to as "memory-dependence". Any adiabatic approximation (e.g., ALDA) utilizes only the instantaneous density, so ignore this temporal nonlocality of the xc potential. Yet, adiabatic approximations work remarkably well for the calculation of most, but not all, excitations and their oscillator strengths. For dynamics in strong fields, there are both parameter regimes and phenomena in which the memory-dependence is minimal, but also examples where memory-dependence is vital $[8,9,33]$. Memory-dependence is not easy to capture in approximations although there has been intense progress recently [34, 36, 38-40].

Ref. [35] used a numerically exactly-solvable example to demonstrate explicitly a case where the correlation potential depends on the density ultra-nonlocally in time. There, time slices were found in which the density was practically identical, semi-locally in time, whereas the correlation potential differed significantly. We shall revisit this example here, with a view to asking whether functionals in a PSDFT could be less non-local in time, and thus easier to approximate. Specifically, we shall ask whether the phase-space density can distinguish those time slices mentioned above, that the coordinate-space density could not. We shall find that indeed it can, and moreover, that the size of the differences in the phasespace density tracks the difference in the correlation potential. This suggests that the memory-dependence in a PSDFT could be milder than in the usual DFT.

Time-dependent Hooke's atom is a system of two 
Coulombically interacting electrons in a time-dependent parabolic well: the Hamiltonian is

$$
\hat{H}=-\frac{1}{2} \nabla_{1}^{2}-\nabla_{2}^{2}+\frac{1}{2} k(t)\left(r_{1}^{2}+r_{2}^{2}\right)+\frac{1}{\left|\mathbf{r}_{1}-\mathbf{r}_{\mathbf{2}}\right|}
$$

For our investigations, we chose $k(t)=\bar{k}-\epsilon \cos (\omega t)$, as in Ref. [35]. The Hamiltonian decouples into relative $(u)$ and center of mass $(R)$ co-ordinates, so one may solve for the exact time-dependent 2-electron wave function as a product of $R$ and $u$ wavefunctions, $\Psi\left(r_{1}, r_{2}, t\right)=$ $\chi(R, t) \xi(u, t)$ and thereby obtain time-dependent density $n(\mathbf{r}, t)$. Knowledge of the time-evolution of the density yields the doubly-occupied time-dependent KS orbital, and then, via inversion of the time-dependent KS equation, the exchange-correlation potential $v_{x c}(\mathbf{r}, t)$. A detailed derivation can be found in Refs. [35, 79], along with computational details. We mention here only that the time-propagation method used is the Crank-Nicholson scheme, and a grid chosen to sample points near the origin more densely than further away, since the density decays exponentially. With chosen parameters $\epsilon=0.1$, $\omega=0.75$, and $\bar{k}=0.25$, the density retains a nearGaussian shape at all times, with a time-varying width; thus it may be parametrized by its variance $r_{r m s}(t)=$ $\sqrt{\left\langle r^{2}\right\rangle}$ [35]. This is shown in the top panel of the Fig. 4. The lower panel shows a density-weighted correlation potential, $\dot{E}_{\mathrm{C}}=\int d^{3} r \dot{n}(\mathbf{r}, t) v_{\mathrm{C}}(\mathbf{r}, t)$, which was also studied in the earlier paper [35], as a simple way to track the time-evolution of the correlation potential. A pair of time slices is indicated within which $r_{r m s}$ is practically identical in each time slice, while the density-weighted correlation potential $\dot{E}_{\mathrm{C}}$ differs quite dramatically. Other such pairs of time slices may be found. This indicates that local, or even semi-local in time density-dependence is not enough to specify the correlation potential, i.e. that the correlation potential depends on a significant history of the density $[9,35]$. Any adiabatic approximation that uses only instantaneous density information would erroneously predict the same correlation potential for each time slice.

We now consider the phase-space distribution at these time slices: if these are also nearly identical at these pairs of time slices, then it would suggest the memorydependence in a PSDFT is just as non-local as in DFT and just as challenging to model. We shall instead find that the phase-space distributions distinguish the system at these different pairs of time slices, with the differences greater with greater differences in the density-weighted correlation potential $\dot{E}_{\mathrm{C}}$. This suggests that perhaps the memory-dependence would be less severe in PSDFT, and therefore perhaps easier to approximate.

Knowledge of the true and KS wavefunctions, enables us to derive expressions for Wigner phase-space distribu-

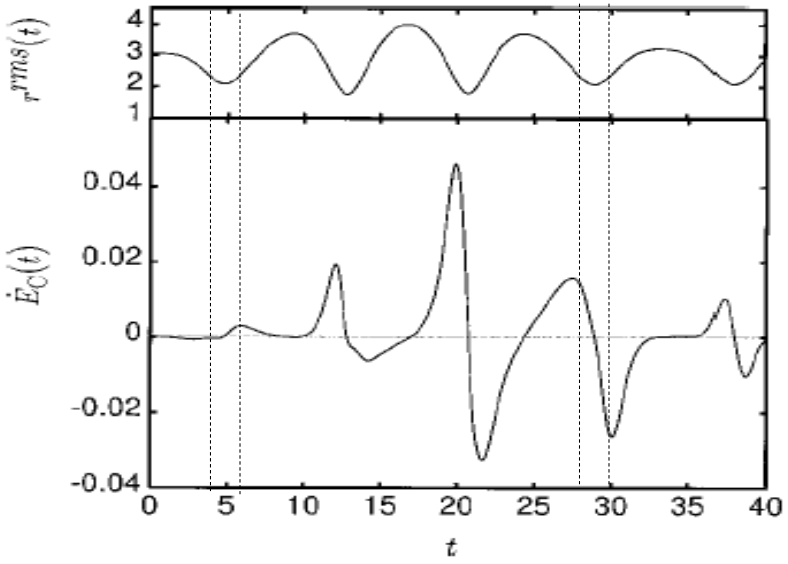

FIG. 4: Nonlocality of the correlation potential in time: $\int v_{\mathrm{C}}(r, t) \dot{n}(r, t) d^{3} r$ (lower panel) and $r_{r m s}(t)$ (top panel). In the two time slices indicated, the density is almost identical locally in time, whereas the density-weighted correlation potential in the lower panel is quite distinct.

tion for the interacting and KS cases:

$$
\begin{gathered}
w(\mathbf{r}, \mathbf{p}, t)=\mid \int_{0}^{\infty} x^{2} d x \int_{-1}^{+1} e^{-i p x \cos \alpha \cos \theta} \\
\times\left. J_{0}\left(p x \sin \alpha \sqrt{1-\cos ^{2} \theta}\right) \chi^{*}\left(\frac{1}{2} \sqrt{r_{+}}, t\right) \xi\left(\sqrt{r_{-}}, t\right) d(\cos \theta)\right|^{2}(22) \\
w_{\mathrm{S}}(\mathbf{r}, \mathbf{p}, t)=\int_{0}^{\infty} x^{2} d x \int_{-1}^{+1} e^{-i p x \cos \alpha \cos \theta} \\
\times J_{0}\left(2 p x \sin \alpha \sqrt{1-\cos ^{2} \theta}\right) \phi^{*}\left(\frac{1}{2} \sqrt{r_{+}}, t\right) \phi\left(\sqrt{r_{-}}, t\right) d(\cos \theta(23)
\end{gathered}
$$

where $r_{+}=r^{2}+x^{2}+2 r x \cos \theta, r_{-}=r^{2}+x^{2}-2 r x \cos \theta$ and $\alpha$ is the angle between $\mathbf{r}$ and $\mathbf{p}$ and $J_{0}$ is the Bessel function of zeroth order. In Eq. 23, $\phi$ is the KS orbital. Both distributions $w$ and $w_{\mathrm{S}}$ depend only on the magnitudes of the coordinate and momentum, $r$ and $p$, and the phase-space angle $\alpha$; for simplicity, we look at the angle-averaged quantities:

$$
w_{a v}(r, p, t)=\int_{0}^{\pi} w(r, p, \alpha, t) \sin \alpha d \alpha
$$

Further, to highlight the component of the Wigner function that is due to correlation, we take the difference between the angle-averaged true and KS Wigner functions, defining

$$
w_{\mathrm{C}}(r, p, t)=w_{a v}(r, p, t)-w_{s, a v}(r, p, t)
$$

To simplify the analysis even further, we consider only the momentum-distribution of this correlation component, defined as:

$$
\tilde{n}_{\mathrm{C}}(p, t)=\tilde{n}(p, t)-\tilde{n}_{\mathrm{S}}(p, t)=\int w_{c}(r, p, t) r^{2} d r
$$



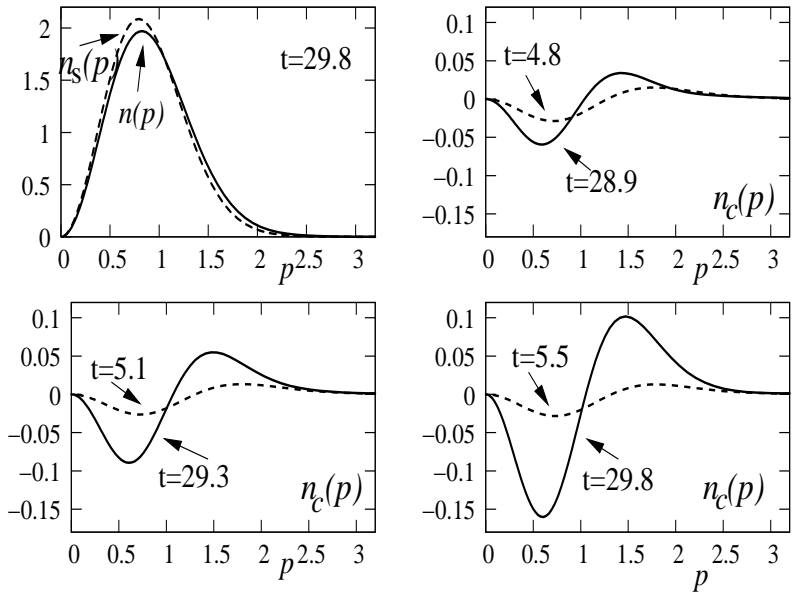

FIG. 5: Top left panel: the true (solid) and KS (dashed) momentum densities, at $t=29.8$. Top right and lower two panels: correlation components of the momentum distributions $n_{\mathrm{C}}(p)$ at three different time-pairs indicated. In each time-pair, the coordinate density, $n(r, t)$, is identical, while the momentum-density is not, with the difference in its correlation component $n_{\mathrm{C}}(p)$ growing as the difference in the density-weighted correlation potential $\dot{E} c$ at those times.

We now turn to the plots in Figure 5. The top left panel shows the true and KS momentum probability distributions at $t=29.8$, which has one of the larger values for the density-weighted correlation potential in this run (Fig. 4). We include in all these plots the Jacobian factor, so that we are actually plotting $p^{2} n(p)$. Although the momentum distributions are not identical, the differences are relatively small, in contrast to the ionization example in the previous section. We now study their difference $n_{\mathrm{C}}(p)$. The other three panels show $n_{\mathrm{C}}(p)$ at pairs of times at which the coordinate-density are practically identical (i.e. essentially indistinguishable to the eye on the scale of plots similar to the top left panel). The first time-pair, $(4.8,29.8)$, is chosen near the minimum of the $r_{r m s}$ at each of the two time slices indicated in Figure 4; here the difference in $\dot{E}_{\mathrm{C}}$ is relatively small $(0.0005 \mathrm{au})$, while it is bigger $(0.011 \mathrm{au})$ at the second time pair $(5.1,29.3)$, and bigger still $(0.0264)$ at the third pair $(5.5,29.3)$. In contrast to the coordinate-density, the momentum-densities are not identical at these pairs of times. Moreover, their difference increases with the difference in the value of the density-weighted correlation potential at those times. The momentum distribution appears to somewhat track the correlation potential.

What are the implications of this for memory in a PSDFT? The results suggest that having momentum information in the basic variable may reduce their memorydependence. Of course this is not conclusive, but the fact that momentum distributions distinguish the system at pairs of times when the coordinate-density does not, yet the correlation potential is different, does suggest that functionals of the phase-space density may be less nonlocal in time.

\section{SUMMARY AND OUTLOOK}

A PSDFT approach could be more successful than the usual TDDFT for some applications for which the usual functional approximations in TDDFT perform poorly. In many strong-field applications, memory-dependence is required in the TDDFT xc potential, challenging to model, and additional observable functionals are needed, depending on the measured quantity of interest. The examples worked through in section III demonstrate each of these aspects clearly. Being exactly-numerically solvable two-electron problems, one can perform a detailed analysis that helps to understand these challenges in their broader context.

The first example, a model of ionization, illustrated, via a phase-space exploration, that even though an exact KS treatment gets the exact coordinate-space density correct, it may do so with a wavefunction that is fundamentally different than the true wavefunction; therefore approximating observables not directly related to the coordinate density by their value on the $\mathrm{KS}$ orbitals does not work well. We considered the momentum distribution (eg modelling that as measured by an ion-momentum recoil experiment), obtained from the KS orbitals and found spurious oscillations as a consequence of the single-determinantal nature of the KS wavefunction: a single delocalized orbital is describing the true two-orbital state. The exact coordinate-space density is recovered (by definition), but the momentum distribution displays oscillations, present also in the KS phasespace distribution. The latter goes negative, a sign of non-classical behavior: the KS system describes the exact density putting both electrons in a doubly-occupied orbital, which must therefore be delocalized in two regions. The true system is however a rather classical one, aside from the antisymmetry, and this is reflected in its phase-space density profile.

The second example explored the memory-dependence of the correlation potential in a time-dependent Hooke's atom. Solving directly for (a density-weighted measure of) the correlation potential, time slices were found in which the coordinate-density evolved identically, while the correlation potential varied dramatically, illustrating the fact that the correlation potential is a highly nonlocal in time functional of the coordinate-density. We then asked whether, if momentum-distribution information was included in the basic variable, the correlation potential would be just as non-local in time as a functional of the phase-space density, or whether the phasespace density could distinguish the state of the system at these times. We found the latter was true, suggesting that correlation functionals of the phase-space density are less non-local in time, and therefore easier to approximate than correlation functionals of the density alone.

In PSDFT, the basic variable is the one-body Wigner phase-space density, from which momentum-space and position-space densities can be obtained via integration over the conjugate variable. Formally equivalent to 
density-matrix functional theory, all one-body observables are directly obtained from the basic variable (including the momentum distribution), without the need for additional observable functionals. In its equation of motion the action of the kinetic energy operator is exact as a phase-space functional (equally, density-matrix functional), while approximations are needed for the secondorder Wigner function as a functional of the first-order one. An important future direction is to develop func- tional approximations for this that lead to changing occupation numbers, in order to treat many of the problems that are challenging in the usual TDDFT, such as electronic quantum control problems, and ionization dynamics. In light of the studies in this paper, this would be well worth pursuing.

We gratefully acknowledge financial support from the National Science Foundation NSF CHE-0547913, and a Research Corporation Cottrell Scholar Award (NTM).
[1] E. Runge and E. K. U. Gross, Phys. Rev. Lett. 52, 997 (1984).

[2] E.K.U. Gross, J.F. Dobson, and M. Petersilka, Topics in Current Chemistry, 181, 81 (1996).

[3] Time-Dependent Density Functional Theory eds. M.A.L. Marques, F. Nogueira, A.Rubio, K.Burke, C.A. Ullrich, and E.K.U. Gross (Springer, Berlin, 2006)

[4] P. Hohenberg and W. Kohn, Phys. Rev. 136, B864 (1964).

[5] M. Petersilka, U.J. Gossmann, and E.K.U. Gross, Phys. Rev. Lett. 76, 1212 (1996).

[6] M.E. Casida, in Recent developments and applications in density functional theory, ed. J.M. Seminario (Elsevier, Amsterdam, 1996).

[7] N. T. Maitra and K. Burke, Phys. Rev. A 63042501 (2001)

[8] N.T. Maitra, K. Burke, and, C. Woodward, Phys. Rev. Lett. 89023002 (2002)

[9] N. T. Maitra, Chapter 4 in Ref. [3]

[10] F. Furche and R. Ahlrichs, J. Chem. Phys. 117 (2002), 7433; J. Chem. Phys. 121 (2004), 12772 (E).

[11] N. Spallanzani, C. A. Rozzi, D. Varsano, T. Baruah, M. Pederson, F. Manghi A. Rubio, arXiv:0901.3052v1 [condmat.mtrl-si]

[12] M. A. L. Marques, X. Lopez, D. Varsano, A. Castro, and A. Rubio, Phys. Rev. Lett. 90, 258101 (2003).

[13] E. Tapavicza, I. Tavernelli, U. Roethlisberger, C. Filippi, M. E. Casida, J. Chem. Phys., J. Chem. Phys. 129, 124108 (2008)

[14] M. Koentopp, C. Chang, K. Burke, and R. Car, J. Phys. Cond. Matt. 20083203 (2008).

[15] D.J. Tozer and N.C. Handy, Phys. Chem. Chem. Phys. 2, 2117 (2000).

[16] N.T. Maitra, J. Chem. Phys. 122, 234104 (2005).

[17] N.T. Maitra, F. Zhang, R.J. Cave and K. Burke, J. Chem. Phys. 120, 5932 (2004).

[18] G. Mazur and R. Wlodarczyk, J. Comp. Chem. (2008).

[19] O. Gritsenko and E.J. Baerends, J. Chem. Phys. 121 655, (2004).

[20] G. Vignale and W. Kohn, Phys. Rev. Lett. 77, 2037 (1996).

[21] S. Kümmel, L. Kronik, and J. P. Perdew, Phys. Rev. Lett. 93, 213002 (200 4).

[22] M. van Faassen, P.L. de Boeij, R. van Leeuwen, J.A. Berger, and J.G. Snijders, Phys. Rev. Lett. 88, 186401 (2002).

[23] J. Parker, L.R. Moore, D. Dnudas, and K.T. Taylor, J. Phys. B: At. Mol. Opt. Phys 33, L691 (2000).

[24] R.Baer, D. Neuhauser, P.R. Zdanska and N. Moiseyev, Phys Rev A 68, 043406 (2003).
[25] H. S. Nguyen, A. D. Bandrauk, and C. A. Ullrich, Phys. Rev. A 69, 063415 (2004)

[26] X. Chu and S. I. Chu, Phys. Rev. A 64, 063404 (2001)

[27] F. Wilken and D. Bauer, Phys. Rev. Lett. 97, 203001 (2006)

[28] D.G. Lappas and R. van Leeuwen, J. Phys. B. 31, L249 (1998).

[29] M. Lein and S. Kmmel, Phys. Rev. Lett. 94, 143003 (2005).

[30] J. P. Perdew and Y. Wang, Phys. Rev. B. 45, 13244 (1992).

[31] F. Wilken and D. Bauer, Phys. Rev. A. 76, 023409 (2007)

[32] M. Thiele, E.K.U. Gross and S. Kmmel, Phys. Rev. Lett. 100, 153004 (2008)

[33] C. A. Ullrich, J. Chem. Phys. 125, 234108 (2006)

[34] C. A. Ullrich and I. Tokatly, Phys. Rev. B.73, 235102 (2006).

[35] P. Hessler, N. T. Maitra, and K. Burke, J. Chem. Phys. 117, 72 (2002).

[36] J.F. Dobson, M.J. Bünner and E.K.U. Gross, Phys. Rev. Lett. 79, 1905 (1997).

[37] Time-dependent density functional theory beyond the adiabatic local density approximation, G. Vignale, C.A. Ullrich, and S. Conti, Phys. Rev. Lett. 79, 4878 (1997).

[38] I.V. Tokatly, Quantum many-body dynamics in a Lagrangian frame: II. Geometric formulation of time dependent density functional theory, Phys. Rev. B 71, 165105 ( 2005).

[39] I.V. Tokatly, Phys. Rev. B 71, 165104 ( 2005).

[40] Y. Kurzweil and R. Baer, Phys. Rev. B. 72, 035106 (2005).

[41] D. G. Tempel, T. J. Martínez, and N.T. Maitra, J. Chem. Theory and Computation, to appear, (2009).

[42] M. E. Casida, F. Gutierrez, J. Guan, F-X. Gadea, D. Salahub, and J-P. Daudey, J. Chem. Phys. 113, 7062 (2000).

[43] O.V. Gritsenko, R. van Leeuwen and E.J. Baerends, J. Chem. Phys. 104, 8535 (1996)

[44] S. Chelkowski, G. L. Yudin, A. D. Bandrauk, J. Phys. B39, S409 (2006).

[45] K. J. H. Giesbertz, E. J. Baerends, O. V. Gritsenko, Phys. Rev. Lett. 101, 033004 (2008).

[46] K. Pernal, O. V. Gritsenko, E. J. Baerends, Phys. Rev. A 75, 012506 (2007).

[47] K. Pernal, K. Giesbertz, O. V. Gritsenko, E. J. Baerends, J. Chem. Phys. 127, 214101 (2007).

[48] S. K. Ghosh and R. G. Parr, Phys. Rev. A 34, 785 (1986).

[49] R. G. Parr, K. Rupnik, and S. K. Ghosh, Phys. Rev. Lett, 56 1555, (1986). 
[50] S. K. Ghosh, M. Berkowitz, and R. G. Parr, Proc. Nat. Acad. Sci. U.S.A. 81, 8028 (1984).

[51] P. M. W. Gill, D. L. Crittendon, D. P. O'Neill, N. A. Besley, Phys. Chem. Chem. Phys. 8, 15 (2006).

[52] D.L. Crittenden, E. E. Dumont, ad P.M.W. Gill, J. Chem. Phys. 127, 141103 (2007).

[53] D.L. Crittenden, P.M.W. Gill, J. Chem. Phys. 127, 014101 (2007).

[54] V. A. Rassolov, J. Chem. Phys. 110, 3672 (1999).

[55] P. Carruthers and F. Zachariasen, Rev. Mod. Phys. 55, 245 (1983).

[56] M. Hillery,R.F. O'Connell, M.O. Scully, and E.P.Wigner, Phys. Rep. 106, 123 (1984)

[57] E. Wigner, Phys. Rev. 40, 749 (1932)

[58] R. W. Spekkens, Phys. Rev. Lett. 101, 020401 (2008).

[59] C. Jacoboni, Rep. Prog. Phys. 67, 1033 (2004).

[60] E. Cancellieri, P. Bordone, A. Bertoni, G. Ferrari and C. Jacoboni, J. Comp. Elec. 3, 411 (2004)

[61] E. Cancellieri, P. Bordone, and C. Jacoboni, Phys. Rev. B. 76, 195317 (2007).

[62] T. L. Gilbert, Phys. Rev. B 12, 2111 (1975).

[63] S.K. Ghosh and A.K. Dhara, Phys. Rev. A 38, 1149 (1988).

[64] E.K.U. Gross and W. Kohn, Adv. Quant. Chem. 21, 255 (1990).

[65] N.T. Maitra, I. Souza, and K. Burke, Phys. Rev. B 68, 045019 (2003).
[66] M. A. Buijse and E.J. Baerends, Mol. Physics. 100, 401 (2002).

[67] S. Sharma, J.K. Dewhurst, N.N. Lathiotakis and E.K.U. Gross, Phys. Rev. B 78, 201103 (2008).

[68] H. Appel and E.K.U. Gross, arXiv:0807.2712v1 (2008).

[69] H. Appel, Time Dependent Quantum Many-Body Systems, Doctoral Thesis, Freie University, Berlin (2007); http://www.diss.fu-berlin.de/2007/481/

[70] K-J. Schmitt, P-G. Reinhard, and C. Toepffer, Z. Phys. A, 336, 1433 (1990).

[71] W. Cassing and A. Pfitzner, Z. Phys. A342, 161 (1992).

[72] M. K. Harbola, R. R. Zope, A. Kshirsagar, and R. K. Pathak, J. Chem. Phys. 122, 204110 (2005).

[73] L. Lam and P. M. Platzman, Phys. Rev. B 9, 5128 (1974); Phys. Rev. B 9, 5122 (1974)

[74] S. Hamel, P. Duffy, M.E. Casida, and D.R. Salahub, J. Electr. Spectr. and Related Phenomena 123, 345 (2002).

[75] P. Duffy, D. Chong, M. E. Casida, and D. R. Salahub, Phys. Rev. A 50, 4707 (1994).

[76] O.V. Gritsenko and E.J. Baerends, Theor. Chem. Acc. 96, 44 (1997).

[77] R.M. Dreizler and E.K.U. Gross, Density Functional Theory, (Springer-Verlag, Berlin, 1990).

[78] G. A. Henderson, Phys. Rev. A 23, 19 (1981).

[79] P. Hessler, J. Park, and K. Burke, Phys. Rev. Lett. 82, 378 (199) 